\title{
Medidas de segurança adotadas nos ambientes hospitalares para o enfrentamento ao COVID-19
}

\author{
Safety measures adopted in hospital environments to face COVID-19 \\ Medidas de seguridad adoptadas en ambientes hospitales para enfrentar el COVID-19
}

\section{Resumo}

A COVID-19 é uma infecção respiratória provocada pelo Coronavírus, Síndrome Respiratória Aguda Grave 2 (SARSCoV-2). A doença é transmitida através de gotículas respiratórias, tosse, espirros, aperto de mão, objetos e superfícies. Objetivo: identificar as medidas de segurança adotada no ambiente hospitalar para o enfretamento da COVID-19. Método: Trata-se de uma revisão integrativa com abordagem qualitativa, método que permite incorporar as pesquisas, utilizando artigos dos anos 2019 a 2021, nas bibliotecas BVS e bases de dados LILACS, MEDLINE, SCIELO, utilizando os descritores (COVID-19), (Hospitals), (Security measures). Resultados e discussões: Nos artigos analisados, observou-se que os hospitais se readaptaram para conseguir enfrentar a nova pandemia obtendo medidas de prevenção como a reorganização nos atendimentos, ampliação dos leitos de unidade de terapia intensa, aumento no abastecimento dos equipamentos de proteção individual, como as máscaras e aventais de proteção e aquisição de teste suficiente para o diagnóstico, criando também, protocolos e estratégias quanto ao uso dos EPIs e higienização das mãos. Considerações finais: relatam que medidas adotadas nos ambientes hospitalares foram a mudanças dos atendimentos, ampliação dos leitos de terapia intensa, aumento dos equipamentos de proteção individual (EPIs) e aquisição de mais testes para os diagnósticos.

Palavras-chave: Pandemia; Ambiente hospitalar; EPI's.

\begin{abstract}
COVID-19 is a respiratory infection caused by Coronavirus, Severe Acute Respiratory Syndrome 2 (SARS-CoV-2). The disease is transmitted through respiratory droplets, coughing, sneezing, shaking hands, objects and surfaces. Objective: to identify the safety measures adopted in the hospital environment to deal with COVID-19. Method: This is an integrative review with a qualitative approach, a method that allows incorporating research, using articles from the years 2019 to 2021, in the VHL libraries and LILACS, MEDLINE, SCIELO databases, using the descriptors (COVID-19), (Hospitals), (Security measures). Results and discussions: In the analyzed articles, it was observed that hospitals were readapted to be able to face the new pandemic, obtaining prevention measures such as the reorganization of care, expansion of intensive care unit beds, increased supply of personal protective equipment, such as masks and protective gowns and acquisition of sufficient test for diagnosis, also creating protocols and strategies regarding the use of PPE and hand hygiene. Final considerations: they report that measures adopted in hospital environments were changes in care, expansion of intensive therapy beds, increase in personal protective equipment (PPE) and acquisition of more tests for diagnosis.
\end{abstract}

Keywords: Pandemic; Hospital Environment; PPE's.

\section{Resumen}

COVID-19 es una infección respiratoria causada por coronavirus, síndrome respiratorio agudo severo 2 (SARS-CoV2). La enfermedad se transmite a través de gotitas respiratorias, toser, estornudar, dar la mano, objetos y superficies. El objetivo de este artículo es identificar las medidas de seguridad adoptadas en el entorno hospitalario para hacer frente al COVID-19. Se trata de una revisión integradora con enfoque cualitativo, método que permite incorporar investigaciones, utilizando artículos de los años 2019 a 2021, en las bibliotecas BVS y bases de datos LILACS, 
MEDLINE, SCIELO, utilizando los descriptores (COVID-19), (Hospitales), (Medidas de seguridad). En los resultados y discusiones de los artículos analizados, se observó que los hospitales se readaptaron para poder enfrentar la nueva pandemia, obteniendo medidas de prevención como la reorganización de la atención, ampliación de camas de la unidad de cuidados intensivos, aumento de la oferta de equipos de protección personal, como mascarillas y batas protectoras y obtendrán pruebas suficientes para el diagnóstico, creando también protocolos y estrategias en cuanto al uso de EPP y separación de guiones en las manos. Las consideraciones finales informan que las medidas adoptadas en los ambientes hospitalarios fueron cambios en la atención, ampliación de camas de terapia intensiva, aumento de equipos de protección personal (EPP) y se obtendrán más pruebas para el diagnóstico.

Palabras clave: Pandemia; Entorno Hospitalario; EPI.

\section{Introdução}

No escritório da Organização Mundial da Saúde (OMS) da China em 31 de dezembro de 2019, foi comunicado casos de pneumonia de etiologia desconhecida detectada na cidade de Wuhan em Província de Hubei. Em 9 de janeiro de 2020, a OMS divulgou um comunicado sobre o conjunto de casos, que afirmou que as autoridades chinesas fizeram uma preliminar determinação de um novo coronavírus, detectado em uma pessoa internada com pneumonia na cidade de Wuhan. O vírus foi inicialmente referido como 2019-COV, mas desde então foi renomeado como SARS-COV-2 pela OMS em 12 fevereiro de 2020 (David \& Koh, 2020).

A doença COVID-19 é uma infecção respiratória provocada pelo Coronavírus da Síndrome Respiratória Aguda Grave 2 (SARS-CoV-2) (Pereira et al., 2020). A transmissão do vírus ocorre de uma pessoa doente para outra, por meio de gotículas respiratórias, espirro, tosse, aperto de mão e por objetos ou superfícies contaminadas, como por exemplo: celulares, maçanetas, mesas e teclado de computador (Cavalcante \& Abreu, 2020).

Os primeiros sintomas do COVID-19 foram confirmados em dezembro de 2019. No começo a morbidade permaneceu baixa, porém atingiu um ponto crítico logo em seguida e o número de pacientes infectados aumentou. Um mês depois a OMS confirmou que havia mais de 10.000 casos de COVID-19 em toda a China. Em fevereiro o número de casos aumentou gradativamente, e atingindo proporções globais (Jiang et al., 2020).

Já no Brasil, o primeiro caso de COVID-19 foi confirmado no dia 26 de fevereiro de 2020. E foi diagnosticada em um idoso residente do estado de São Paulo que havia retornado de uma viagem da Itália, com isso a doença se propagou rapidamente. Até o final do mês de março foram confirmados mais de 5.700 casos de COVID-19, com mais de 200 óbitos. No entanto, 2.750.318 casos com 94.665 óbitos foram confirmados até 01 de agosto (Marinelli et al., 2020).

Nesse contexto, o serviço de saúde deparou-se com a formação de um novo cenário em relação às ações em saúde e segurança dos profissionais. Portanto, os ambientes hospitalares obtiveram mudanças e reavaliação dos protocolos para prevenção dos trabalhadores expostos ao vírus durante seus trabalhos laborais (Silva et al., 2020).

Os profissionais da saúde encontram-se no grupo de risco para a COVID-19, pois ficam expostos diretamente aos pacientes infectados, e com isso recebem uma alta carga viral. Assim proteção da saúde dos profissionais e pacientes é fundamental para evitar transmissão de COVID-19 nos ambientes hospitalares e nas suas próprias residências, sendo necessário adotar protocolos de controle de infecções e disponibilizar EPIs (Teixeira et al., 2020).

Para a prevenção máxima de segurança contra a transmissão da doença, conforme o Regulamento Sanitário Internacional (RSI), foi adotado algumas medidas para minimizar seus efeitos e reduzir os casos de morbidade e mortalidade causada pelo COVID-19 (Camargo et al., 2020).

Os equipamentos de proteção individuais (EPIs) têm como função a barreira contra o agente patógeno na região da face, das mãos, dos olhos e outras partes do corpo. Os EPIs são roupas e aparatos usados para proteção contra riscos à saúde e segurança no trabalho. Máscaras, capotes, respiradores, luvas, óculos de proteção, protetores faciais, aventais e revestimentos para cabeça e calçados são os mais utilizados (Assunção et al., 2020).

As mãos tornam-se um dos principais meios de contágio ao tocar em pessoas contaminadas e superfícies, com isso 
deve ser frequentemente higienizada para evitar a propagação do vírus. A higienização por meio da lavagem constante e de maneira correta com água e sabão e/ ou a utilização de antisséptico, principalmente soluções etanólicas ou isopropílicas, que são produto/ substancias mais recomendados (Sequinel et al., 2020).

Diante o exposto, a presente pesquisa tem como objetivo identificar as medidas de segurança adotada no ambiente hospitalar para o enfretamento da COVID-19.

\section{Metodologia}

Trata-se de uma revisão integrativa com abordagem qualitativa, método que permite incorporar as pesquisas realizadas em áreas da saúde e da educação, emerge como uma metodologia que proporciona a síntese do conhecimento e a incorporação da aplicabilidade de resultados de estudos significativos na prática (Sousa et al., 2018).

A pesquisa foi elaborada em cinco fases: Determinação da pergunta norteadora e dos objetivos; coleta de dados, análise crítica dos estudos incluídos na pesquisa; discussão dos resultados e revisão integrativa.

A busca de estudos ocorreu na biblioteca BVS e bases de dados LILACS, MEDLINE, SCIELO. Utilizaram-se descritores para localizar os artigos, com coerência para evitar possíveis desvios de assunto. Os descritores utilizados foram: "COVID-19", "hospitals" e "Security measures", com o auxílio do booleano "AND".

As buscas abrangeram artigos sobre a temática e publicados entre dezembro de 2019 a março de 2021. Para o desenvolvimento da pesquisa foram utilizando os critérios de seleção para artigos completos, inglês, português e espanhol, como critérios de exclusão, artigos que não tivessem relacionados com o tema abordado, artigos não completos e artigos que não se encontrava disponíveis gratuitamente nas bases de dados.

A seleção dos artigos foi realizada seguindo a estratégia PRISMA (Preferred Reporting Items for Sistematic Reiews and Meta-analyses), conforme demonstração da Figura 1.

Figura 1 - Fluxograma do processo de seleção dos artigos para revisão integrativa de literatura, elaborado com base nas recomendações PRISMA.

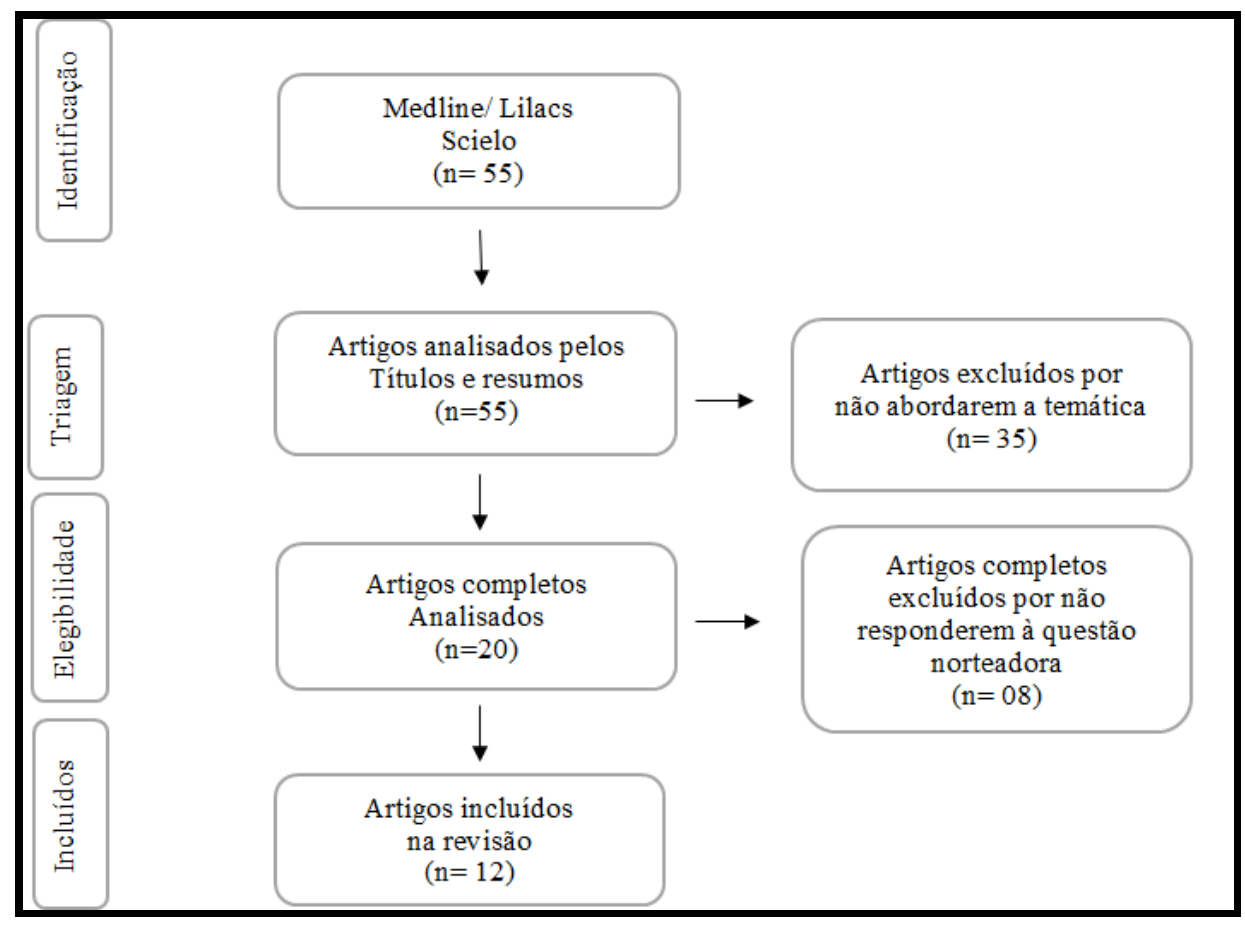

Fonte: Autores. 
A Figura 1 retrata como ocorreu o processo de seleção dos artigos que foram utilizados no estudo, dos 55 artigos encontrados na plataforma somente 12 artigos atenderam aos critérios estabelecidos.

\section{Resultados e Discussão}

Foi encontrado um total de 55 artigos. Após a leitura de títulos e resumos foram excluídos 35 por não corresponderem ao tema do estudo. Os 20 artigos restantes foram analisados na integra e doze foram selecionados, já que respondiam à pergunta norteadora. Oito artigos foram excluídos por não responderem à questão norteadora.

A compilação sintética dos doze artigos selecionados para o desenvolvimento da pesquisa com referência, títulos, objetivos e resultados constam no Quadro 1.

Quadro 1 - Artigos sobre as medidas de segurança adotadas nos hospitais para o enfretamento ao COVID-19

\begin{tabular}{|c|c|c|c|}
\hline Autor/ ano & Títulos & Objetivos & Resultados \\
\hline $\begin{array}{c}\text { Zang } \\
(2020)\end{array}$ et $\quad$ al. & $\begin{array}{l}\text { Status de proteção ocupacional } \\
\text { no } \\
\text { 19 Fangcang Shelter Hospital } \\
\text { em Wuhan, China. }\end{array}$ & $\begin{array}{l}\text { Analisar o pessoal e o status de } \\
\text { proteção ambiental do } \\
\text { Lago Fangcang Leste-Oeste } \\
\text { Hospital Abrigo }\end{array}$ & $\begin{array}{l}\text { Por meio de treinamento e supervisão } \\
\text { cuidadosa, a maioria deles era competente para } \\
\text { trabalhar nas áreas de internação seis horas / } \\
\text { dia, três a quatro vezes / semana. Além disso, } \\
7,8 \% \text { vivenciaram diversos tipos de exposição } \\
\text { ocupacional, causados principalmente por } \\
\text { danos de EPI. }\end{array}$ \\
\hline $\begin{array}{c}\text { Silva } \\
(2020)\end{array}$ & $\begin{array}{l}\text { Atuação do serviço de controle } \\
\text { de infecção hospitalar em } \\
\text { hospital público da paraíba no } \\
\text { enfrentamento à COVID-19: } \\
\text { relato de experiência. }\end{array}$ & $\begin{array}{l}\text { Descrever a atuação do Serviço } \\
\text { de Controle de Infecção } \\
\text { Hospitalar (SCIH) do Hospital } \\
\text { Metropolitano diante da } \\
\text { pandemia da COVID-19. }\end{array}$ & $\begin{array}{l}\text { Sendo possível salientar que a SCIH está } \\
\text { atuando desde os primeiros casos de SRAG e } \\
\text { COVID-19 admitidos na instituição de forma } \\
\text { dinâmica e presencial realizando ações para } \\
\text { minimizar a propagação do vírus no hospital e } \\
\text { manter a segurança dos pacientes e da equipe } \\
\text { de saúde. }\end{array}$ \\
\hline $\begin{array}{l}\text { Silva et al. } \\
(2020)\end{array}$ & $\begin{array}{l}\text { Segurança do trabalho no } \\
\text { ambiente hospitalar frente à } \\
\text { pandemia da COVID-19 }\end{array}$ & $\begin{array}{l}\text { Identificar trabalhos científicos } \\
\text { que abordem as medidas de } \\
\text { segurança do trabalho no } \\
\text { ambiente hospitalar frente à } \\
\text { pandemia do novo coronavírus. }\end{array}$ & $\begin{array}{l}\text { Foram selecionados seis estudos, publicados } \\
\text { em 2020, indexados nas bases MEDLINE, } \\
\text { LILACS e BDENF. Todos se tratavam de } \\
\text { relatos de casos/experiência. Apenas um deles } \\
\text { foi produzido por enfermeiros e os outros por } \\
\text { médicos. Quanto aos países de origem dos } \\
\text { artigos, pode-se citar: Brasil, Itália, Turquia, } \\
\text { Austrália e República da Coréia. }\end{array}$ \\
\hline $\begin{array}{l}\text { Marques et al. } \\
(2020)\end{array}$ & $\begin{array}{l}\text { COVID-19: cuidados de } \\
\text { enfermagem para segurança no } \\
\text { atendimento de serviço pré- } \\
\text { hospitalar móvel. }\end{array}$ & $\begin{array}{l}\text { Descrever as ações realizadas } \\
\text { por enfermeiros do serviço pré- } \\
\text { hospitalar móvel antes, durante e } \\
\text { após atendimentos e } \\
\text { transferências de pacientes } \\
\text { suspeitos e/ou confirmados para } \\
\text { COVID-19 e as limitações } \\
\text { encontradas por esses } \\
\text { profissionais para diminuir a } \\
\text { exposição à doença. }\end{array}$ & $\begin{array}{l}\text { O estudo permitiu refletir sobre a } \\
\text { multidimensionalidade de ações necessárias } \\
\text { para prevenção e controle da pandemia. Foram } \\
\text { identificadas condutas para garantir a segurança } \\
\text { instrumental nas unidades móveis, a segurança } \\
\text { profissional e a segurança do paciente em } \\
\text { atendimento pré-hospitalar móvel. }\end{array}$ \\
\hline $\begin{array}{l}\text { Branco et al. } \\
(2020)\end{array}$ & $\begin{array}{l}\text { Serviço de emergência } \\
\text { hospitalar: fluxos de } \\
\text { atendimento a pacientes } \\
\text { suspeitos ou confirmados para } \\
\text { COVID-19. }\end{array}$ & $\begin{array}{l}\text { Relatar a experiência vivenciada } \\
\text { de um serviço de emergência } \\
\text { hospitalar do Sistema Único de } \\
\text { Saúde (SUS) sobre os fluxos de } \\
\text { atendimento a pacientes } \\
\text { suspeitos ou confirmados por } \\
\text { COVID-19. }\end{array}$ & $\begin{array}{l}\text { Para atendimento dos pacientes suspeitos ou } \\
\text { confirmados de infecção por coronavírus, } \\
\text { foram organizados planos de contingência e } \\
\text { fluxos de atendimento nos setores de } \\
\text { emergência em conexão com as demais áreas } \\
\text { do hospital. Criaram-se o Centro de Triagem e } \\
\text { a sala vermelha COVID-19. }\end{array}$ \\
\hline $\begin{array}{l}\text { Oliveira et al. } \\
(2020)\end{array}$ & $\begin{array}{l}\text { Equipamento de proteção } \\
\text { individual na pandemia por } \\
\text { coronavírus: treinamento com } \\
\text { prática deliberada em ciclos } \\
\text { rápidos }\end{array}$ & $\begin{array}{l}\text { Discutir a aplicação da Prática } \\
\text { Deliberada em Ciclos Rápidos } \\
\text { para o treinamento de } \\
\text { paramentação } \\
\text { desparamentação no contexto da } \\
\text { COVID-19 e estruturar um guia } \\
\text { prático para a aplicação nesta } \\
\text { conjuntura. }\end{array}$ & $\begin{array}{l}\text { Maximização do tempo em Prática } \\
\text { Deliberada, feedback com evidência e } \\
\text { segurança psicológica são os princípios desta } \\
\text { estratégia. A dinâmica envolve repetições } \\
\text { e feedbacks. O guia de aplicação apresenta a } \\
\text { sequência de ações para paramentação e } \\
\text { desparamentação. }\end{array}$ \\
\hline
\end{tabular}




\begin{tabular}{|c|c|c|c|}
\hline $\begin{array}{lll}\text { Batista } & \text { et } & a l . \\
(2020) & & \end{array}$ & $\begin{array}{l}\text { Estratégia multimodal para } \\
\text { higiene das mãos em hospitais } \\
\text { de campanha de COVID- } 19 .\end{array}$ & 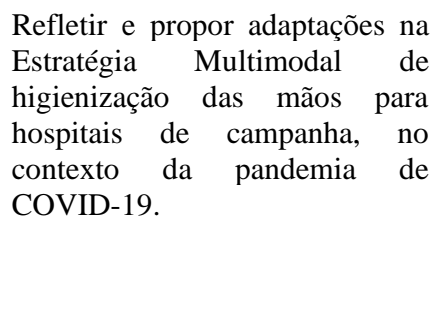 & $\begin{array}{l}\text { A Estratégia Multimodal, proposta para } \\
\text { hospitais em geral, pode ser adaptada para } \\
\text { hospitais de campanha visando reduzir a } \\
\text { transmissão do vírus SARSCoV-2. } \\
\text { Investimentos para adequar a infraestrutura e } \\
\text { educação de trabalhadores exigem previsão e } \\
\text { celeridade e são de especial relevância para } \\
\text { promover a higienização das mãos nesse } \\
\text { contexto assistencial. }\end{array}$ \\
\hline $\begin{array}{l}\text { Cunha et al. } \\
(2020)\end{array}$ & $\begin{array}{l}\text { Atuação da equipe } \\
\text { multiprofissional em saúde, no } \\
\text { cenário da pandemia por } \\
\text { COVID -19. }\end{array}$ & $\begin{array}{l}\text { Apresentar reflexão teórica } \\
\text { acerca da abordagem da equipe } \\
\text { multiprofissional em Nefrologia } \\
\text { no cenário do COVID-19. }\end{array}$ & $\begin{array}{l}\text { O novo coronavírus apresenta alta } \\
\text { transmissibilidade e, por isso, é necessário } \\
\text { implementar medidas de prevenção e controle, } \\
\text { que contam com a capacitação de uma equipe } \\
\text { multiprofissional no estabelecimento das } \\
\text { estratégias a serem seguidas e orientadas. }\end{array}$ \\
\hline $\begin{array}{l}\text { Helioterio et al. } \\
(2020)\end{array}$ & $\begin{array}{l}\text { COVID-19: por que a proteção de } \\
\text { trabalhadores e trabalhadoras da } \\
\text { saúde é prioritária no combate à } \\
\text { pandemia? }\end{array}$ & $\begin{array}{l}\text { Objetiva-se sumarizar e } \\
\text { sistematizar aspectos relativos às } \\
\text { condições de trabalho e de saúde } \\
\text { dos(as) trabalhadores(as) da } \\
\text { saúde nessa pandemia, } \\
\text { enfatizando a situação no Brasil, } \\
\text { experiências exitosas na } \\
\text { proteção do trabalho em saúde } \\
\text { em outros países e } \\
\text { recomendações para o contexto } \\
\text { brasileiro. }\end{array}$ & $\begin{array}{l}\text { Para o enfrentamento da COVID-19 no Brasil, } \\
\text { destacam-se como recomendações: revisão de } \\
\text { fluxos de atendimento e definição de } \\
\text { características e condições para cada etapa de } \\
\text { atendimento; estabelecimento da COVID-19 } \\
\text { como doença relacionada ao trabalho para os } \\
\text { grupos expostos; registro efetivo da 'ocupação' } \\
\text { nos sistemas de informação; estabelecimento de } \\
\text { condições especiais para execução do trabalho } \\
\text { na situação de epidemia; atenção às jornadas } \\
\text { laborais e ações para redução de estressores } \\
\text { ocupacionais. }\end{array}$ \\
\hline $\begin{array}{l}\text { Locks et al. } \\
(2020)\end{array}$ & $\begin{array}{l}\text { Qualidade da higienização das } \\
\text { mãos de profissionais atuantes em } \\
\text { unidades básicas de saúde }\end{array}$ & 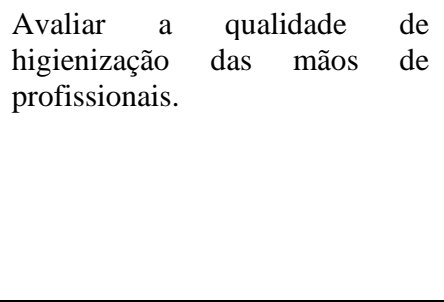 & $\begin{array}{l}\text { Enquanto nos procedimentos clínicos o } \\
\text { percentual foi } 31,7 \% \text {. Constatou-se que, } \\
\text { durante as cirurgias, os profissionais de nível } \\
\text { superior realizaram melhor a higienização das } \\
\text { mãos do que os de nível médio }(\mathrm{p}<0,05) \text { e, nos } \\
\text { procedimentos clínicos, os profissionais de } \\
\text { idade superior a } 35 \text { anos apresentaram melhor } \\
\text { desempenho do que os mais moços }(\mathrm{p}<0,05) \text {. }\end{array}$ \\
\hline Medeiros (2020) & $\begin{array}{l}\text { Desafios para o enfrentamento da } \\
\text { pandemia COVID-19 em } \\
\text { hospitais universitários }\end{array}$ & $\begin{array}{l}\text { Os hospitais universitários } \\
\text { brasileiros, centros de formação } \\
\text { e qualificação profissional, bem } \\
\text { como de produção de } \\
\text { conhecimento, têm significativo } \\
\text { papel no enfrentamento dessa } \\
\text { epidemia. }\end{array}$ & $\begin{array}{l}\text { Como profissionais de saúde, temos de nos } \\
\text { preparar para o pior nas próximas semanas, nos } \\
\text { proteger, ter esperança e estar na linha de } \\
\text { frente, contribuindo nessa importante luta } \\
\text { histórica contra esse novo coronavírus. }\end{array}$ \\
\hline $\begin{array}{l}\text { Boszczowsk } \\
(2021)\end{array}$ & $\begin{array}{l}\text { Comissões de controle de } \\
\text { infecção hospitalar em tempos } \\
\text { de pandemia }\end{array}$ & $\begin{array}{l}\text { Propusemos a esboçar uma breve } \\
\text { reconstituição da sequência de } \\
\text { eventos vivida pelos } \\
\text { profissionais de serviços de } \\
\text { controle de infecção no Brasil e } \\
\text { sua necessidade de rápida e } \\
\text { constante adaptação, ao mesmo } \\
\text { tempo em que havia absorção de } \\
\text { novos conhecimentos em ritmo } \\
\text { acelerado e pressões geradas } \\
\text { pela opinião pública. }\end{array}$ & $\begin{array}{l}\text { O caminho de volta à operação usual dos } \\
\text { serviços de saúde, e hospitalar em particular, é } \\
\text { uma fase com muitos desafios. O primeiro é } \\
\text { manter o nível de atenção e vigilância dos } \\
\text { profissionais e pacientes que frequentam o } \\
\text { serviço, de modo a garantir a manutenção das } \\
\text { medidas de prevenção. A percepção de que as } \\
\text { coisas voltar à normalidade pode reduzir o } \\
\text { estado de alerta e levar a práticas inseguras. O } \\
\text { segundo aspecto é monitorar o volume dos } \\
\text { atendimentos "não COVID-19", uma vez que o } \\
\text { "represamento" da demanda poderá determinar } \\
\text { aumento imprevisível e nova sobrecarga aos } \\
\text { serviços de saúde. }\end{array}$ \\
\hline
\end{tabular}

Fonte: Dados do estudo (2021).

Após a análise minuciosa dos artigos, pode-se identificar a evidencia que o ambiente hospitalar precisou passar por uma adaptação para enfrentar a COVID -19, com ação de protocolos com relação ao uso de equipamentos de proteção individual (EPIs), treinamento para os profissionais da saúde quanto ao uso correto dos EPIs, estratégias para higienização das 
mãos e uso de antissépticos.

De acordo com Medeiros (2020) e Branco et al. (2020) o grande desafio para os hospitais foram reorganizar os atendimentos para que todos pudessem se prevenir do vírus. Com isso os atendimentos dos pacientes exigiram uma restruturação imediata, bem como construir novos planejamentos baseados na experiência hospitalar, desenhando assistência de todo o serviço emergencial e atendimentos separados de acordo com o nível de infecção. Os autores relataram que foi necessário que os hospitais ampliassem os leitos de unidade de terapia intensa, aumento de abastecimento dos equipamentos de proteção individual, como as máscaras e aventais de proteção e teste suficiente para o diagnóstico.

Em dois estudos os ambientes hospitalares obtiveram novo protocolo para a proteção dos profissionais e pacientes. O protocolo teve como função regularizar o uso de EPIs estabelecendo a utilização de acordo com o risco de transmissão de infecções, ou seja, para cada tipo de assistência aos pacientes os profissionais de saúde eram orientados quanto ao uso dos EPIs e quais usarem (Silva et al., 2020). Outro estudo apontou que os requisitos para o uso dos EPIs eram estabelecidos de acordo com protocolo de nível de biossegurança, por exemplo, pessoal de sucção de amostragem do trato respiratório e o pessoal de análise de ácido nucleico e sangue, foram protegidos no nível de biossegurança 3, como: mascaras N95, protetores faciais, capotes e luvas (Zhang et al., 2020).

Segundo a estratégia de higienização das mãos Batista et al. (2020), ressalta que é interessante utilizar a estratégia multimodal, uma ferramenta de implementação para melhoria do processo de cuidado e resultados dos pacientes de uma maneira estruturada, tem como objetivo melhorar a higiene das mãos pelos profissionais da saúde, através das ações propostas pela estratégia, para diminuição das ocorrências de infecções por COVID-19, mostrando para todos a forma correta de higienizar as mãos. Para hospitais a Estratégia Multimodal é válida e aplicável para contribuir com a prática assistencial nessas unidades assistenciais.

Nos estudos de Marques et al. (2020), ressalta orientação para higienização das mãos com água e sabão preferencialmente, utilizando as técnicas adequada de higienização, como: movimentos em toda palma das mãos e dos dedos, friccionando separadamente os polegares, pontas dos dedos e punho. De acordo com Locks et al. (2020) a outra maneira de higienizar as mãos é com antissépticos, que consta com a limpeza das mãos e em seguida a utilização de um produto antisséptico, com função de remover microrganismos, fazendo com que diminua a carga de microbiana das mãos. O ministério da saúde recomenda como produtos antissépticos o álcool 70, a clorexidina e triclosan. Os autores citados concordam que as medidas adotadas para a redução da propagação do vírus seja a higienizar corretas das mãos e uso de antissépticos.

De acordo com Silva et al. (2020) e Oliveira et al. (2020) os profissionais da saúde foram treinando para manusear corretamente os EPIs, pois a infecção por COVID-19 durante o surto de emergência, ocorreu, principalmente, nos profissionais da saúde. O treinamento sobre o uso adequado dos EPIs torna-se imprescindível e eficaz, pois fazendo o manejo e tendo todos os cuidados com os pacientes infectados, a contaminação dos profissionais é reduzida. Mas Sousa et al. (2020) ressalta que apesar dos profissionais serem treinados para usar os EPIs, eles estão se contaminando com a desparamentação inadequadas, por exemplo, a manipulação das máscaras com as mãos contaminadas.

As máscaras faciais tornam-se eficazes no combate da proliferação do vírus. De acordo com Cunha et al. (2020), existe vários tipos de as máscaras, cirúrgicas N95, N100, PFF3, PFF2, entre outras, sendo classificadas em dois tipos, a que retém partículas solidas e líquidos a base de água e óleo e a resistente ao aerossol que retém partículas solidas e liquidas a base de água. Segundo ANVISA as duas máscaras mais utilizadas na prática são a N95 e a PFF2. As máscaras classificadas PFF2 seguem as normas brasileiras ABNT/NBR 13698:2011 e ABNT/NBR 13697:2010 e a norma europeia apresenta eficiência filtração de 94\%. Já as máscaras N95 seguem a norma americana e apresentam eficiência filtração de 95\%. Ambos são considerados equivalentes (ANVISA, 2020).

Dessa forma, dois estudos relatam que foram criadas intervenções estratégicas e protocolos de prevenção aos 
profissionais da saúde para combater a pandemia. Os pontos estratégicos foram elencar as condições de trabalho, onde fornece aquisições dos EPIs bem como orientações do uso correto, redução das jornadas de trabalho e novos fluxos de atendimento nos serviços de saúde (Helioterio et al., 2020). Os protocolos obtiveram como orientação a higienização das mãos, relato imediato em caso de sintomas respiratórios, avaliação clínica e testagem de profissionais sintomáticos e garantia de disponibilidade de equipamentos de proteção individual (Boszczowsk, 2021).

\section{Considerações Finais}

No presente estudo, foram sistematizados conhecimentos sobre as medidas de prevenção adotadas dentro de um ambiente hospitalar para o enfretamento ao COVID-19. Em suma, compreendemos que os ambientes hospitalares sofreram surtos com o coronavírus e com isso tiveram que se readaptar a novos protocolos. Observa-se que as medidas adotadas nos hospitais foram a mudanças dos atendimentos, ampliação dos leitos de terapia intensiva, aumento dos equipamentos de proteção individual (EPIs) e aquisição mais testes para os diagnósticos. Logo, programaram estratégias para a higienização das mãos e uso de antissépticos. Dessa forma os profissionais da saúde foram treinados novamente quanto ao uso dos EPIs, quais seriam utilizados e a forma correta de se usar de acordo com o nível de infecção, com isso a contaminação seria reduzida.

Espera-se que os profissionais da saúde continuem conscientes da importância paramentação adequada, assim como a fiscalização por parte da instituição e ofereçam cursos de capacitação para os funcionários. Entretanto é necessário salientar que o estudo em questão abre caminhos para a construção de futuros trabalhos, direcionado ao entendimento do uso de equipamento de proteção individual e coletiva no meio hospitalar, que reduza a transmissão da COVID-19 entre paciente para profissional.

\section{Referências}

ANVISA. (2021) Agência Nacional de Vigilância Sanitária. Nota Técnica No 04/2020 GVIMS / GGTES/ANVISA. Orientações para serviços de saúde: medidas de prevenção e controle que devem ser adotadas durante a assistência aos casos suspeitos ou confirmados de infecção pelo novo coronavírus (SARSCoV-2). 118. https://www.gov.br/anvisa/pt-br/centraisdeconteudo/publicacoes/servicosdesaude/notas-tecnicas/nota-tecnica-gvims_ggtes_anvisa-04_2020-2502-para-o-site.pdf

Assunção, A., Simões, M., Maia, E., Alcântara, M. \& Jardim, R. (2020). COVID-19: Limites na implantação dos equipamentos de proteção individuais recomendados aos profissionais de saúde. Scientific Electronic Library Online, (1): 1-16. https://doi.org/10.1590/SciELOPreprints.1018

Batista J., Silva D. P., Nazário S. S. \& Cruz E. D. A. (2020). Multimodal strategy for hand hygiene in field hospitals of COVID-19. Revista Brasileira Enfermagem, 73(2), 1-6. https://doi.org/10.1590/0034-7167-2020-0487

Boszczowski, I. (2020). Comissões de controle de infecção hospitalar em tempos de pandemia. Acesso e cuidados especializado, 148 . https://docs.bvsalud.org/biblioref/2021/03/1150769/COVID-19-volume5.pdf\#page=149

Branco, A., Milanes, R., Sakamoto, V. T. M., Araújo, B. R. \& Caregnato, R. C. A. (2020). Serviço de emergência hospitalar: fluxos de atendimento a pacientes suspeitos ou confirmados para COVID-19. Enfermagem em Foco, 11(1), 199-204. http://biblioteca.cofen.gov.br/wpcontent/uploads/2020/09/servico-emergencia-hospitalar-sus-atendimento-pacientes-COVID-19.pdf

Camargo, M. C. D., Martinez, S., Lima, A. A., Bastos, B. P., Santos, D. L. D., Mota, S. E. D. C. \& Toledo, I. P. D. (2020). Eficácia da máscara facial (TNT) na população para a prevenção de infecções por coronavírus: revisão sistemática. Ciência \& Saúde Coletiva, 25, 3365-3376. https://doi.org/10.1590/141381232020259.13622020

Cavalcante, J. R. \& Abreu, A. D. J. L. D. (2020). COVID-19 no município do Rio de Janeiro: análise espacial da ocorrência dos primeiros casos e óbitos confirmados. Epidemiologia e Serviços de Saúde, 29(3), 1-10. https://doi.org/10.5123/S1679-49742020000300007

Cunha, T. G. S., Guimarães, A. S. M., Santos, T. A. \& Freire, L. B. V. (2020). Atuação da equipe multiprofissional em saúde, no cenário da pandemia por Covid 19. Health Residencies Journal-HRJ, 1(1), 1-22. https://doi.org/10.51723/hrj.v1i2.37

Helioterio, M. C., Lopes, F. Q. R. D. S., Sousa, C. C. D., Souza, F. D. O., Pinho, P. D. S., Nogueira, F. \& Sousa, F. D. (2020). COVID-19: por que a proteção da saúde dos trabalhadores e trabalhadoras da saúde é prioritária no combate à pandemia? Trabalho, Educação e Saúde, 18(3). https://repositorio.unb.br/handle/10482/37953

Koh, D. (2020). Occupational risks for COVID-19 infection. Occupational medicine, 70(1), 1-10. https://www.ncbi.nlm.nih.gov/pmc/articles/PMC7107962/

Locks, L., Lacerda, J. T., Gomes, E. \& Tine, A. C. P. S. (2020). Qualidade da higienização das mãos de profissionais atuantes em unidades básicas de saúde. Revista Gaúcha de Enfermagem, 32(3), 569-575. https://doi.org/10.1590/S1983-14472011000300019 
Research, Society and Development, v. 10, n. 8, e57710817587, 2021

(CC BY 4.0) | ISSN 2525-3409 | DOI: http://dx.doi.org/10.33448/rsd-v10i8.17587

Marinelli, N. P., Albuquerque, L. P. D. A., Sousa, I. D. B. D., Batista, F. M. D. A., Mascarenhas, M. D. M. \& Rodrigues, M. T. P. (2020). Evolução de indicadores e capacidade de atendimento no início da epidemia de COVID-19 no Nordeste do Brasil, 2020. Epidemiologia e Serviços de Saúde, 29. https://doi.org/10.5123/S1679-49742020000300008

Medeiros, E. A. S. (2020). Desafios para o enfrentamento da pandemia COVID-19 em hospitais universitários. Revista Paulista de Pediatria, 38. https://doi.org/10.1590/1984-0462/2020/38/2020086

Oliveira, H. C., Souza, L. C. D., Leite, T.C. \& Campos, J. F. (2020). Equipamento de Proteção Individual na pandemia por coronavírus: treinamento com Prática Deliberada em Ciclos Rápidos. Revista Brasileira Enfermagem, 73. https://doi.org/10.1590/0034-7167-2020-0303

Pereira, M. D., Oliveira, L. C. D., Costa, C. F. T., Bezerra, C. M. D. O., Pereira, M. D., Santos, C. K. A. D. \& Dantas, E. H. M. (2020). A pandemia de COVID-19, o isolamento social, consequências na saúde mental e estratégias de enfrentamento: uma revisão integrativa. Research, Society and Development, 9(7), 80-93. https://doi.org/10.1590/0034-7167-2020-0303

Silva, A. D. S., Santos, G. D., Teodózio, G. C. \& Cordeiro, K. J. D. S. (2021). Atuação do serviço de controle de infecção hospitalar em hospital público da Paraíba no enfrentamento à COVID-19: relato de experiência. Environmental smoke, 4(1), 32-43. https://doi.org/10.32435/envsmoke.20214132-43

Silva, R. C. D. M., Silva, M. C. D. M. \& Costa, C. R. B. (2020). Segurança do trabalho no ambiente hospitalar frente à pandemia da COVID-19. Revista de Atenção à Saúde, 18(65), 29-39. https//doi.org/10.13037/ras.vol18n65.7071

Sousa, J. G. M. D., Ribeiro, W. A., Franco, A. D.A., Floriano, A. D. A., Sousa, A. B. T., Carvalho, B. L., Guinancio, J.C. \& Dias, L. L. C. (2021). Utilização dos equipamentos de proteção individual a partir da ótica da equipe de enfermagem no enfrentamento do COVID-19. Recima21-Revista Científica multidisciplinar, 2(4), 24225-24231. https://doi.org/10.47820/recima21.v2i4.225

Teixeira, C. F. S., Soares, C. M., Souza, E. A., Lisboa, E. S., Pinto, I. C. M. Andrade, L. R. \& Espiridião, M. A. (2020). A saúde dos profissionais de saúde no enfrentamento da pandemia de COVID-19. Ciência \& Saúde Coletiva, 25, 3465-3474. https://doi.org/10.1590/1413-81232020259.19562020

Zhang, M., Wang, L., Yu, S. \& Sun, G. (2020). Status of occupational protection in the COVID-19 Fangcang Shelter Hospital in Wuhan, China. Emerging microbes \& infections, 9(1), 1835-1842. https://doi.org/10.1080/22221751.2020.1803145 